\title{
New Transformation for Efficient DSP Control of Active Power-Line Conditioners
}

\author{
L. Cheng, R. Cheung \\ Ryerson Polytechnic University \\ Department of Electrical \& Computer Engineering \\ 350 Victoria Street \\ Toronto, Ontario, Canada M5B 2K3 \\ email: Icheng@ee.ryerson.ca
}

\begin{abstract}
This paper presents a new transformation scheme for the control of active power-line conditioner. An active powerline conditioner is a power electronic device which is designed to suppress the power-line distortions caused by the non-linear loads. Currently, the method of instantaneous power transformation is the most popular one for the active power-line conditioning control. This method uses the measurements of the power-line voltage and current to estimate the required harmonic compensation. The method requires many hardware for the measurements and many computations. The high accuracy hardware for measurements are costly and many computations can introduce truncation errors. Also, this method is sensitive to the power-line voltage measurements and may introduce control errors [1]. A new simple transformation method is developed to overcome these difficulties. This control computes the correct harmonic compensation using the power-line current measurement only. This requires less hardware for the power-line voltages measurements and simplifies the computational processes. Unlike the traditional method, the accuracy of the new control method does not affected by the line voltage measurements, therefore, it is reliable under any power-line condition. An active power-line conditioner has been successfully developed to validate the accuracy and efficiency of the new control method. Typical test results are given in the paper.
\end{abstract}

\section{Introduction}

An active power-line conditioner provides a viable solution to the power-line harmonic interference problems and it can be used to protect the sensitive devices from harmonic distortions [2-7]. Solid-state power electronic devices regulate the flow of power by chopping the sinusoidal input current which introduces harmonic disturbances into the supply system. These disturbances

\author{
R. Sotudeh \\ University of Teesside \\ School of Science \& Technology \\ Teesside, Middlesbrough, UK TS1 3BA
}

circulates the power system and can cause serious process failure to the neighbouring devices. The active power-line conditioner monitors the current harmonics and produces a compensating current accordingly for current harmonic cancellations $[2,3]$. The standard method for monitoring the current harmonics uses the instantaneous power approach [2,7-11]. It uses the line voltage and current to compute the instantaneous power with respect to $\alpha \beta$ axis. This instantaneous power consists of $\mathrm{dc}$ components and ac components. The dc components represent the fundamental $60-\mathrm{Hz}$ current and the ac components represents the current harmonics. The compensating current can be estimated by the inverse transformation of the ac components. The new transformation method simplified the computation processes and is easier to be implemented using the state-of-the-art digital signal processor (DSP) for the computation processes. This new method uses the power-line current measurement only and a sine function table. The measured line current is multiplied to a sine function at the fundamental $60-\mathrm{Hz}$ and transformed the with rotating frame. The transformed signals consist of dc components and ac components. The $\mathrm{dc}$ components represent the fundamental $60-\mathrm{Hz}$ current and ac components represent the current harmonics even the line voltage is distorted. An inverse transformation of the ac components is the required compensating current.

This paper uses simulations and an experimental results to illustrate the effectiveness of the active power-line conditioner. The power-line conditioner with the new transformation method for the control uses less hardware and can suppress the current harmonics effectively.

\section{Review of Instantaneous Power Method}

The instantaneous power method uses the line voltage and current measurements to estimate the compensating current. First, the line voltage $\left(V_{a}, V_{b}, V_{c}\right)$ and current $\left(I_{a}\right.$, $I_{b}, I_{c}$ ) measurements are transformed into $\alpha \beta$ reference by 
multiplying the transformation matrix $\mathbf{C}$ as below [2]:

$$
C=\sqrt{\frac{2}{3}}\left[\begin{array}{ccc}
1 & \frac{-1}{2} & \frac{-1}{2} \\
0 & \frac{\sqrt{3}}{2} & \frac{-\sqrt{3}}{2}
\end{array}\right]
$$

Therefore, the three phase line voltage and current with respect to $\alpha \beta$ axis become:

$$
\begin{aligned}
& {\left[\begin{array}{c}
V_{a} \\
V_{\beta}
\end{array}\right]=C *\left[\begin{array}{l}
V_{a} \\
V_{b} \\
V_{c}
\end{array}\right]} \\
& {\left[\begin{array}{c}
I_{\alpha} \\
I_{\beta}
\end{array}\right]=C *\left[\begin{array}{l}
I_{a} \\
I_{b} \\
I_{c}
\end{array}\right]}
\end{aligned}
$$

Second, the transformed voltage $\left(\mathrm{V}_{\alpha}, \mathrm{V}_{\beta}\right)$ and current $\left(I_{\alpha}, I_{\beta}\right)$ are used to compute the instantaneous power by using the equation as below:

$$
\left[\begin{array}{l}
p \\
q
\end{array}\right]=\left[\begin{array}{cc}
v_{\alpha} & v_{\alpha} \\
-v_{\beta} & v_{\alpha}
\end{array}\right]\left[\begin{array}{c}
i_{\alpha} \\
i_{\beta}
\end{array}\right]
$$

where $\mathbf{p}$ is the real power and $\mathbf{q}$ is the imaginary power.

Both $\mathbf{p}$ and $\mathbf{q}$ consist of a dc component and an ac components. The dc component is the sum of the product of the fundamental current with the fundamental voltage and the product of $n^{\text {th }}$ order harmonic current with the $n^{\text {th }}$ order harmonic voltage. If the line voltage is sinusoidal, the dc component corresponds to the fundamental $60-\mathrm{Hz}$ current and the ac components correspond to the current harmonics [1]. By eliminating the dc component, the inverse transformation of the ac components are the current harmonics which are as follow:

$$
\left[\begin{array}{c}
i_{h \alpha} \\
i_{h \beta}
\end{array}\right]=\frac{1}{V_{\alpha}^{2}+V_{\beta}^{2}}\left[\begin{array}{cc}
V_{\alpha} & -V_{\beta} \\
V_{\beta} & V_{\alpha}
\end{array}\right]\left[\begin{array}{l}
p_{a c} \\
q_{a c}
\end{array}\right]
$$

where $i_{\text {ho }}$ is the current harmonics in $\alpha$-axis $i_{h \beta}$ is the current harmonics in $\beta$-axis

Consider a simple case of the line currents are distorted. The line-voltages and currents are assumed with respect to $\alpha \beta$ reference as below:

$$
\left[\begin{array}{c}
\mathrm{V}_{\alpha} \\
\mathrm{V}_{\beta}
\end{array}\right]=\sqrt{\frac{3}{2}} \mathrm{~V}_{1}\left[\begin{array}{c}
\sin (\omega \mathrm{t}) \\
-\cos (\omega \mathrm{t})
\end{array}\right]
$$

where $V_{1}$ is the magnitude of the fundamental $60-\mathrm{Hz}$ voltage.

$\left[\begin{array}{l}I_{a} \\ I_{\beta}\end{array}\right]=\sqrt{\frac{3}{2}} I_{1}\left[\begin{array}{c}\sin (\omega t-\gamma)+d \sin (n \omega t-\delta) \\ -\cos (\omega t-\gamma)-d \sin (n \omega t-\delta)\end{array}\right]$

where $I_{1}$ is the magnitude of the fundamental $60-\mathrm{Hz}$ current

$\mathrm{d}$ is the ratio of the current harmonic to the fundamental current

$\gamma$ is the phase shift of the fundament current with respect to the fundament voltage

$\delta$ is the phase shift of the current harmonic with respect to the fundamental voltage

The instantaneous power is computed by using Equation (3) and it is as the following:

$$
\begin{aligned}
& \mathrm{p}=\frac{3}{2} \mathrm{~V}_{1} \mathrm{I}_{1}\{\cos \gamma+\mathrm{d} \cos [(\mathrm{n}-1) \omega \mathrm{t}-\delta]\} \\
& \mathrm{q}=\frac{3}{2} \mathrm{~V}_{1} \mathrm{I}_{1}\{-\sin \gamma+\mathrm{d} \sin [(\mathrm{n}-1) \omega \mathrm{t}-\delta]\}
\end{aligned}
$$

Both $\mathbf{p}$ and $\mathbf{q}$ consist a dc component and many ac components. By eliminating the dc component, the ac components become:

$$
\begin{aligned}
& p_{a c}=\frac{3}{2} V_{1} I_{1}(d \cos [(n-1) \omega t-\delta]) \\
& q_{a c}=\frac{3}{2} V_{1} I_{1}(d \sin [(n-1) \omega t-\delta])
\end{aligned}
$$

By applying the Equation (4), an inverse transformation of the ac components in Equation (8) will give the current harmonics in Equation (9).

$$
\begin{aligned}
& I_{h \alpha}=\sqrt{\frac{2}{3}} I_{1} d \sin (n \omega t-\delta) \\
& I_{h \beta}=-\sqrt{\frac{2}{3}} I_{1} d \cos (n \omega t-\delta)
\end{aligned}
$$

The instantaneous power method uses both the measured power-line voltages and currents to compute the current harmonics. A complete computation process for the current harmonics requires one division, twenty-six multiplications and additions. The instantaneous power method requires many hardware for the measurements and computations. 


\section{New Transformation Method}

The new transformation method is evoluted from the modulating method [12]. This modulating method replaces the power-line voltage with a modulating signal which is defined in $\alpha \beta$ reference $\left(m_{\alpha}, m_{\beta}\right)$ as below:

$$
\begin{aligned}
& m_{\alpha}=\sin (\omega t) \\
& m_{\beta}=-\cos (\omega t)
\end{aligned}
$$

The line current measurements are transformed into $\alpha \beta$ axis by using Equation (1). Then, the modulating signals multiply with the transformed line current and gives the modulated signals (Mp, Mq) which are defined as the following:

$$
\left[\begin{array}{l}
M p \\
M q
\end{array}\right]=\left[\begin{array}{cc}
m_{\alpha} & m_{\beta} \\
-m_{\beta} & m_{\alpha}
\end{array}\right]\left[\begin{array}{c}
I_{\alpha} \\
I_{\beta}
\end{array}\right]
$$

Both Mp and Mq consist of a dc component and many ac components. The ac components represent the current harmonics and the dc component corresponds to the fundamental $60-\mathrm{Hz}$ current. The $\mathrm{dc}$ components of modulated signals are eliminated and the current harmonics are the inverse transformation of the ac components ( $\mathbf{M} \mathbf{p}_{\mathbf{a c}}, \mathbf{M} \mathbf{q}_{\mathbf{a c}}$ ) as below:

$$
\left[\begin{array}{c}
\mathrm{i}_{\mathrm{h} \alpha} \\
\mathrm{i}_{\mathrm{h} \beta}
\end{array}\right]=\left[\begin{array}{cc}
\mathrm{m}_{\alpha} & -\mathrm{m}_{\beta} \\
\mathrm{m}_{\beta} & \mathrm{m}_{\alpha}
\end{array}\right]\left[\begin{array}{c}
M \mathrm{p}_{\mathrm{ac}} \\
\mathrm{Mq}_{\mathrm{ac}}
\end{array}\right]
$$

The new transformation method uses the modulating matrix in Equation (11) to multiply with the matrix $\mathbf{C}$ as shown in Equation (1) and form a new transformation matrix:

$$
\sqrt{\frac{2}{3}}\left[\begin{array}{ccc}
\sin \omega t & \sin \left(\omega t+\frac{4 \pi}{3}\right) & \sin \left(\omega t+\frac{2 \pi}{3}\right) \\
\cos \omega t & \cos \left(\omega t+\frac{4 \pi}{3}\right) & \cos \left(\omega t+\frac{2 \pi}{3}\right)
\end{array}\right] \text { (3) }
$$

where $\omega$ is the fundamental $377-\mathrm{rad} / \mathrm{sec}$.

Similarly, the inverse transformation matrix is the multiplication product of the de-modulating matrix in Equation (12) and the inverse of the matrix $\mathbf{C}$ in Equation (1).
This inverse transformation matrix becomes:

$$
\sqrt{\frac{2}{3}}\left[\begin{array}{cc}
\sin \omega t & \cos \omega t \\
\sin \left(\omega t+\frac{4 \pi}{3}\right) & \cos \left(\omega t+\frac{4 \pi}{3}\right) \\
\sin \left(\omega t+\frac{2 \pi}{3}\right) & \cos \left(\omega t+\frac{2 \pi}{3}\right)
\end{array}\right]
$$

The new transformation method uses Equation (13) to transformed the measured power-line current with respect to a rotating frame at the fundamental $60-\mathrm{Hz}(377-\mathrm{rad} / \mathrm{sec})$. The transformed current contains a dc component and ac component. This dc component represents the fundamental power-line current and the ac component corresponds to the current harmonics. An inverse transformation using the Equation (14) will gives the current harmonics in the time-domain.

Consider that the distorted power-line current is given as:

$$
\left[\begin{array}{l}
I_{a} \\
I_{b} \\
I_{c}
\end{array}\right]=I_{1}\left[\begin{array}{c}
\sin (\omega t-\gamma)+d \sin (n \omega t-\delta) \\
\sin \left(\omega t-\gamma-\frac{2 \pi}{3}\right)+d \sin \left(n \omega t-\delta-\frac{2 \pi}{3}\right) \\
\sin \left(\omega t-\gamma-\frac{4 \pi}{3}\right)+d \sin \left(n \omega t-\delta-\frac{4 \pi}{3}\right)
\end{array}\right]
$$

where $I_{1}$ is the magnitude of the fundamental current $\mathrm{d}$ is the ratio of the current harmonic to the fundamental current $\gamma$ is the phase shift of the fundament current with respect to the transformation frame $\delta$ is the phase shift of the current harmonic with respect to the transformation frame

By transforming this power-line current into the rotating frame using Equation (13), the transformed current $\left(i_{p}, i_{q}\right)$ become:

$$
\left[\begin{array}{l}
i_{p} \\
i_{q}
\end{array}\right]=\sqrt{\frac{3}{2}} I_{1}\left[\begin{array}{c}
\cos \gamma+d \cos [(n-1) \omega t-\delta] \\
-\sin \gamma+d \sin [(n-1) \omega t-\delta]
\end{array}\right]
$$

Both $\mathbf{i}_{\mathrm{p}}$ and $\mathbf{i}_{\mathrm{q}}$ consist of $\mathrm{a} \mathrm{dc}$ component and an ac component. The dc component is eliminated and the ac components become:

$$
\begin{aligned}
& i_{p a c}=\sqrt{\frac{3}{2}} d I_{1} \cos [(n-1) \omega t-\delta] \\
& i_{q a c}=\sqrt{\frac{3}{2}} d I_{1} \sin [(n-1) \omega t-\delta]
\end{aligned}
$$

The current harmonic is the inverse transformation of the 
ac components using Equation (14). The current harmonics is found as:

$$
\left[\begin{array}{l}
i_{h a} \\
i_{h b} \\
i_{h c}
\end{array}\right]=d I_{1}\left[\begin{array}{c}
\sin (n \omega t-\delta) \\
\sin \left(n \omega t-\delta-\frac{2 \pi}{3}\right) \\
\sin \left(n \omega t-\delta-\frac{4 \pi}{3}\right)
\end{array}\right]
$$

The computed current harmonics in Equation (18) is the same as it was assumed in Equation (15). This new transformation method does not require power-line voltage for the compensating current estimation. Therefore, it requires less hardware. Also, the computation processes is less complicated compared to that of using the instantaneous power method. A complete computation process for the current harmonics computation requires only twelve additions and multiplications. Unlike the instantaneous power method, there is no division in the computation process. This new method can easily be implemented using digital signal processor as well as analog circuit.

\section{Simulation}

This simulation illustrates the effectiveness of the instantaneous power method and new modulation method. Figure 1 shows the system configuration used for the simulation. The non-linear load is a typical voltage-source inverter induction motor drive. The active power-line conditioner estimates the required compensating current from the power-line measurements for current injection. This active power-line conditioner is assumed lossless and the switching devices can response instantaneously. The effectiveness of the active power-line conditioner is merely depended on the accuracy of the compensating current estimation of the power-line conditioning control method.

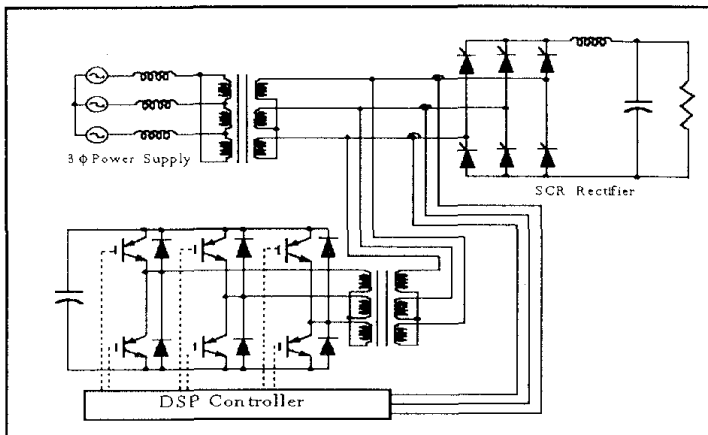

Figure 1 Basic Active Filter System Configuration
Figure 2 shows the typical power-line current waveforms without any harmonic compensation in the input line. It is shown that the line current waveforms of the ac drive are highly distorted. This severe harmonic source is used to test the capability of the conditioner using the new transformation method and the instantaneous power method.

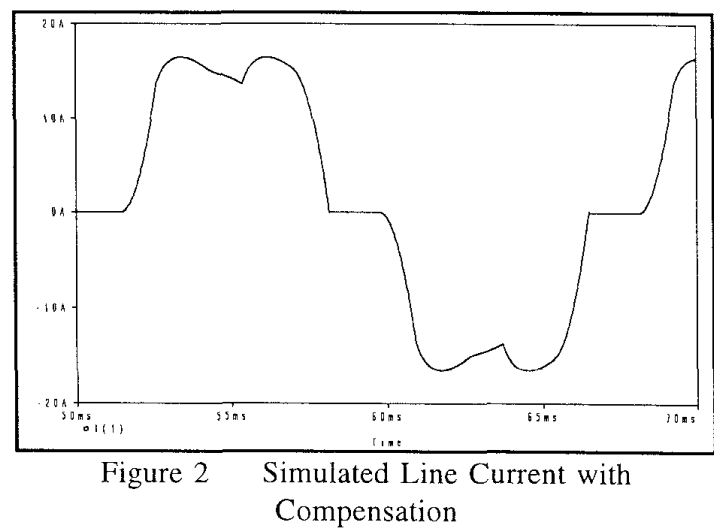

Figure 3 shows the current harmonics estimation using the instantaneous power method and Figure 4 shows the waveforms using the new transformation method.

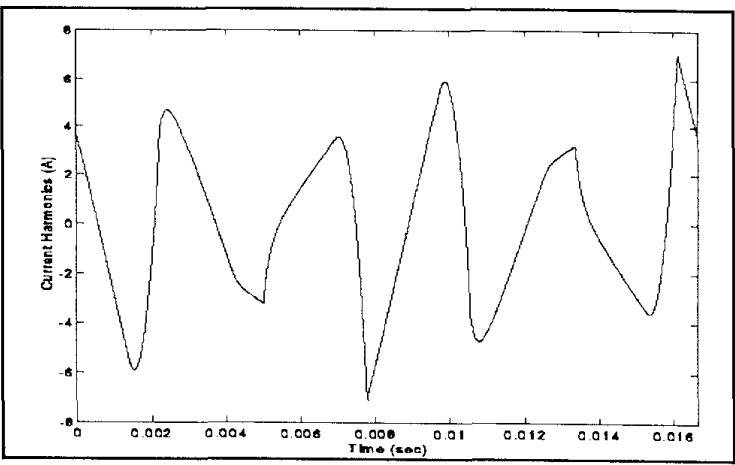

Figure 3 Estimated Current Harmonics Using the Instantaneous Power Method

Computer simulations have showed that the new transformation method can be used to estimate the current harmonics as accurate as the instantaneous power method.

\section{Experimental Results}

A 10-kVA active power-line conditioner has been developed with the new transformation method. Figure 1 shows the basic system configuration for testing the 


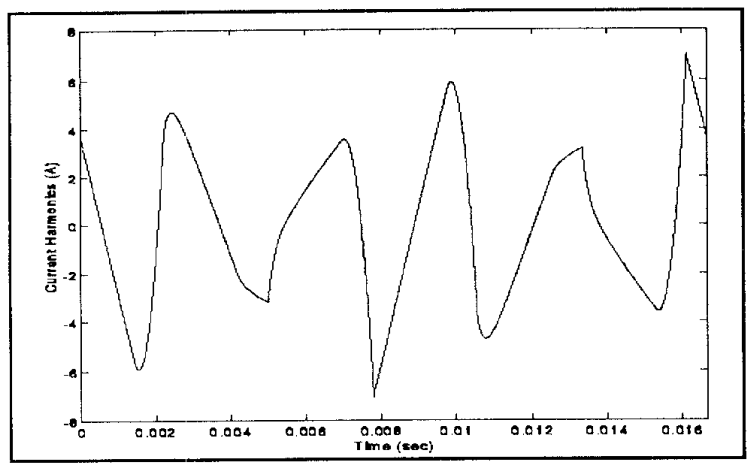

Figure 4 Estimated Current Harmonics Using the New Transformation Method

effectiveness of this power-line conditioner. The nonlinear rectifier load is used to produce the current harmonics and pollute the power-line. The active powerline conditioner uses the state-of-the-art digital-signal processor to implement the new transformation control method. This digital-signal processor uses the measured power-line current and multiplies to the sine function table in the memory and gives the transformed current. By eliminating the average transformed current which represented the dc component of the transformed current, an inverse transformation of the remaining ac component will gives the estimated compensating current. This computed compensating current is used to generate the PWM switching signals to the inverter. The inverter turns on/off the switching devices and injects the current for compensation.

Figure 5 shows the typical waveforms measured from the conditioner circuit. In this figure, the first graph is the typical current waveform at the input of a non-linear rectifier load. The second graph is the compensating current waveform injected from the power-line conditioner. The third graph is the compensated power-line current waveform. This graph shows that most of the power-line harmonics are compensated.

\section{Conclusion}

This paper proposes a new transformation method for the control of the active power-line conditioner. This method basically use a similar concept of the standard method. First transform the measured data (current or voltage). Second eliminate the dc component of the transformed data. Third compute the control reference from the

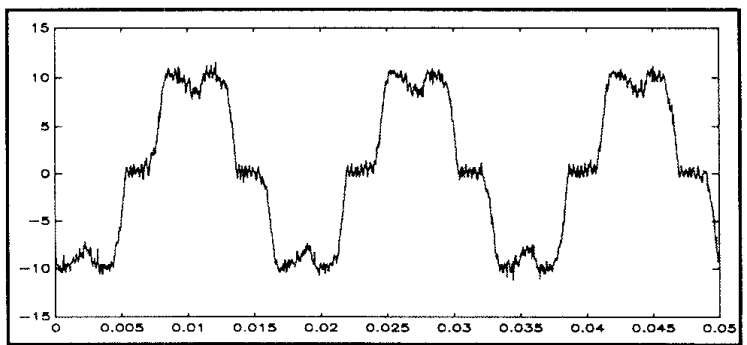

(a) Load current

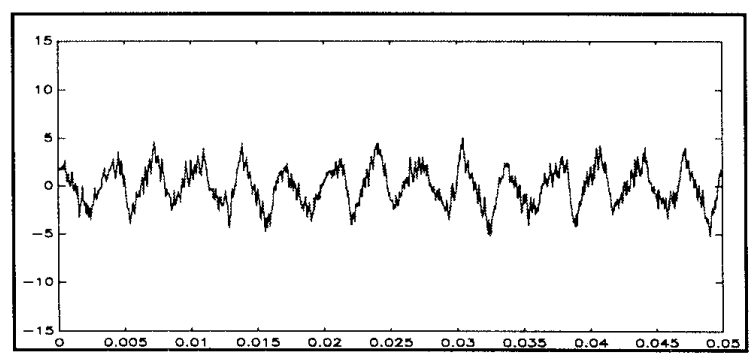

(b) Compensating current

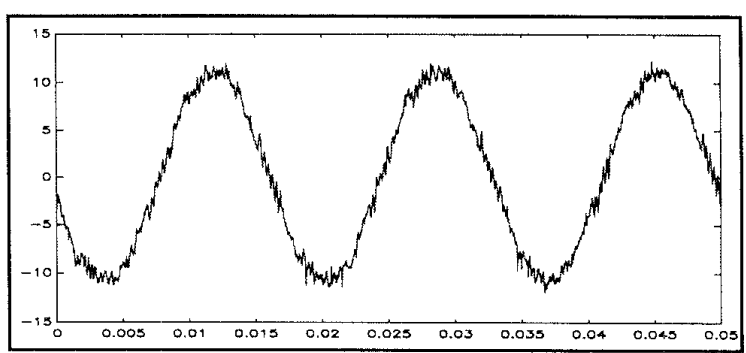

(c) Compensated current

Figure 5 Measured Current Waveforms

remaining ac component. The standard control method requires transformations of both line voltage and line current data to compute the reference for guiding the power-line conditioner operation. The proposed control method simplifies the transformations using the line current only. This provides several practical merits over the standard method such as:

- Simplified Hardware Implementation - The new method requires to process power-line current measurements only. This simplifies substantially the hardware requirements particularly for accurate high-voltage data processing.

Enhanced Control Data Processing - The new method only requires to process the line current measurement, so that the required data processing 
is much simpler and faster. In particular, the new method uses less computational procedures and does not require any numerical division as compared to the standard method.

- Reliable Control under Practical Operation Conditions - The standard method uses the ac component of the instantaneous power to compute the control reference for the power-line conditioners. However, under non-ideal conditions with distorted line voltages, the ac component may not accurately represent the line distortion. This can result in errors in the control reference. The new method process only the line current, and therefore the line voltage distortion has no effect on the resulting control reference.

\section{Reference}

1. Cavallini A. and Montanari G., "Compensation Strategies for Shunt Active-Filter Control," IEEE Trans. on Power Elec., Vol. 9., pp. 587-593, Nov. 1994.

Akagi H., Kanazawa Y. and Nabae A., "Instantaneous Reactive Power Compensators Comprising Switching Devices without Energy Storage Components, "IEEE Trans. on Ind. Appl., Vol IA-20, No. 3, pp. 625-630, May/June 1984

3. Grady W.M., Samotyj M.J. and Noyola A.H., "Survey of Active Power Line Conditioning Methodologies," IEEE Trans. on Power Delivery, Vol. 5, No. 3, July 1990

4. Grady M.W., Samotyj M., Noyola A.H., "Improving Power Quality with Active Power Line Conditioners," First International Conference on Power Quality, Paris France, pp. 213-220, Oct 1991

5. Malesani L., Rosetto L., Tenti P., "Active Filter for Reactive Power and Harmonic Compensation," Proc. of IEEE-PESC, pp. 321 330, June 1986

6. Akagi H., Tuskamoto Y., Nabae A., "Analysis and Design of an Active Power Filter using Quad-Series Voltage-Source PWM Converters," Conf. Rec. of IEEE-IAS Annual Meeting, pp. 1168-1173, Oct 1988
7. Peng F.Z., Akagi H. and Nabae A., "A Study of Active Power Filters Using Quad-Series VoltageSource PWM Converters for Harmonic Compensation," IEEE PESC, pp. 204-212, 1987

8. Fujita H. and Akagi H., "A Practical Approach to Harmonic Compensation in Power Systems Series Connection of Passive and Active Filters," IEEE Trans. Ind. Appl., Vol. 27, No. 6, pp. 1020-1025, Nov/Dec 1991

9. H. Akagi, "Trends in Active Power Line Conditioners," IEEE Power Elec., pp. 263-268, May 1994

10. Malesani L. Rosetto L., Tenti P., "Active Power Filter with Hybrid Energy Storage," IEEE-PESC, pp. 385-391, June 1989

11. J.F. Chicharo, H. Wang, "Power System Harmonic Signal Estimation and Retrieval for Active Power Filter Applications," IEEE Trans. Power Elec., pp. 580-586, Nov. 1994

12. L. Cheng, R. Cheung, R. Sotudeh, "Instantaneous Harmonic Current Compensation Using a Novel Modulation Scheme," UPEC, pp. 121, Sept. 1994 